\title{
Comparison of Gross Body Fat-Water Magnetic Resonance Imaging at 3 Tesla to Dual Energy X-Ray Absorptiometry in Obese Women
}

\author{
HJ Silver ${ }^{1}$, KD Niswender ${ }^{2,3}$, J Kullberg $^{4}$, J Berglund ${ }^{4}$, L Johansson ${ }^{4}$, M Bruvold $^{5}$, MJ \\ Avison $^{6,7}$, and EB. Welch ${ }^{6}$
}

${ }^{1}$ Division of Gastroenterology, Hepatology and Nutrition, Department of Medicine, Vanderbilt University School of Medicine, Nashville, Tennessee ${ }^{2}$ Tennessee Valley Healthcare System, Nashville, Tennessee ${ }^{3}$ Division of Diabetes, Endocrinology \& Metabolism, Department of Medicine, Vanderbilt University School of Medicine, Nashville Tennessee ${ }^{4}$ Department of Radiology, Uppsala University, Uppsala, Sweden ${ }^{5} \mathrm{MR}$ Clinical Science, Philips Healthcare, Best, Netherlands ${ }^{6}$ Vanderbilt University Institute of Imaging Sciences, Department of Radiology and Radiological Sciences, Vanderbilt University School of Medicine, Nashville, Tennessee ${ }^{7}$ Department of Pharmacology, Vanderbilt University School of Medicine, Nashville, Tennessee

\section{Abstract}

\begin{abstract}
Improved understanding of how depot-specific adipose tissue mass predisposes to obesity-related comorbidities could yield new insights into the pathogenesis and treatment of obesity as well as metabolic benefits of weight loss. We hypothesized that three-dimensional contiguous "fat-water" MR imaging (FWMRI) covering the majority of a whole-body field of view (FOV) acquired at 3 Tesla (3T) and coupled with automated segmentation and quantification of amount, type and distribution of adipose and lean soft tissue would show great promise in body composition methodology. Precision of adipose and lean soft tissue measurements in body and trunk regions were assessed for 3T FWMRI and compared to DEXA. Anthropometric, FWMRI and DEXA measurements were obtained in twelve women with BMI $30-39.9 \mathrm{~kg} / \mathrm{m}^{2}$. Test-retest results found coefficients of variation for FWMRI that were all under 3\%: gross body adipose tissue (GBAT) $0.80 \%$, total trunk adipose tissue (TTAT) $2.08 \%$, visceral adipose tissue (VAT) $2.62 \%$, subcutaneous adipose tissue (SAT) $2.11 \%$, gross body lean soft tissue (GBLST) $0.60 \%$, and total trunk lean soft tissue (TTLST) 2.43\%. Concordance correlation coefficients between FWMRI and DEXA were $0.978,0.802,0.629$, and 0.400 for GBAT, TTAT, GBLST and TTLST, respectively. While Bland Altman plots demonstrated agreement between FWMRI and DEXA for GBAT and
\end{abstract}

\footnotetext{
Users may view, print, copy, and download text and data-mine the content in such documents, for the purposes of academic research, subject always to the full Conditions of use:http://www.nature.com/authors/editorial_policies/license.html\#terms

Correspondence: Heidi J. Silver, Department of Medicine, Vanderbilt University, Nashville, TN, 37232-2713,

Heidi.j.silver@vanderbilt.edu, 6159361299 And E. Brian Welch, Department of Radiology and Radiological Sciences, Vanderbilt University, Nashville, TN, 37232-2713, Brian.welch@vanderbilt.edu, 6153437754.

Disclosure

The other authors have no duality of interest to disclose.

Supplementary Material

Supplementary material is available at www.nature.com/obesity
} 
TTAT, a negative bias existed for GBLST and TTLST measurements. Differences may be explained by the FWMRI FOV length and potential for DEXA to overestimate lean soft tissue. While more development is necessary, the described 3T FWMRI method combined with fullyautomated segmentation is fast ( $<30$ minutes total scan and post-processing time), noninvasive, repeatable and cost effective.

\section{Keywords}

Body Composition; Body Fat Distribution; DEXA; Obesity; Magnetic Resonance; Fat Water Imaging

\section{Introduction}

Quantifying the amount, type and distribution of adipose and lean soft tissue is critical to understanding not only the pathological roles of these tissues in the metabolic consequences of obesity but also the metabolic benefits of dietary, pharmacological and surgical weight loss interventions $(1,2)$. While more sophisticated methods for body composition analysis would be applicable to numerous research questions, our research interests are focused on obesity in women as adipose tissue distribution and its metabolic consequences are quite different between women and men. Moreover, as women age, the percentage of body fat and amount of visceral adipose tissue (VAT) increases significantly (3). The increasing adiposity observed over the life cycle in women is an important factor in the lack of achievement of the same successes that have occurred in men with regard to reduced cardiovascular risk over the past few decades. It is conceivable that the gaps in the evidence to explain the biological underpinnings of cardiometabolic risk may be due to the relative strengths and weaknesses of information available from current body composition assessment techniques.

Imaging methods such as dual energy $\mathrm{x}$-ray absorptiometry (DEXA), magnetic resonance imaging (MRI) and computed tomography (CT) are considered the most accurate for quantifying body composition (4). Because DEXA scanners are widely available, they are used most frequently to estimate body composition. However, since DEXA acquires data as a two-dimensional coronal projection it does not distinguish type of fat, e.g., VAT versus subcutaneous adipose tissue (SAT), and it is not able to discern ectopic fat. Further, it has been demonstrated that the error in DEXA derived estimation of total and regional fat mass (typically overestimation) increases in proportion to the amount of body fat mass (adiposity) and abdominal sagittal thickness (5). In addition, the trunk region typically determined using DEXA includes chest, abdomen, pelvis and some gluteal fat, which confounds measures in obese women (6). Even with manipulation of the region of interest, DEXA tends to estimate intra-abdominal adipose tissue best in nonobese persons (7). With regard to lean soft tissue estimation, DEXA has been shown to overestimate skeletal muscle mass, partly due to the effects of hydration status $(8,9)$.

While both CT and MRI provide quantification of tissue volume, MRI is preferred because, unlike CT, it does not expose individuals to ionizing radiation. In fact, the potential of MRI for assessment of amount, type and distribution of adipose tissue has long been recognized (10), although low sensitivity and long scan times have limited widespread usage. In more 
recent investigations, multi-gradient-echo MRI acquisitions have been used with 1.5 Tesla (1.5T) scanners to acquire body composition data sets more quickly than standard MR anatomical imaging protocols (11). However, the use of multi-slice MRI methods with large inter-slice gaps requires interpolation and geometric assumptions to calculate tissue volumes (12). We suggest that such MRI methods could be optimized to sample contiguously almost the entire body with no gap between slices, thus, eliminating the necessity to interpolate data.

In fact, conventional T1-weighted MRI has been validated for quantification of adipose tissue in cadaver studies (13) and used successfully in vivo $(10,12,14)$. However, T1weighted spin-echo or T1-weighted spoiled gradient-echo scans attempt to discriminate adipose tissue versus non-adipose tissues based on signal intensity because the relatively short T1 (longitudinal magnetization relaxation rate time constant) of adipose tissue causes it to be bright on T1-weighted scans. As other tissues such as blood vessels and bowel contents may also appear bright on T1-weighted images, semi-automatic segmentation may be necessary to eliminate segmentation errors. In contrast to T1-weighted imaging, MRIbased chemical shift imaging, termed "fat-water MRI," is molecule-specific and takes advantage of the small but measurable differences in the resonance frequencies of water and lipid protons. With this method, high fat content is more specific to adipose tissue than obtained with short T1. Fat-water MRI can be used to assess body tissues with a threedimensional (no gap between slices) multi-gradient-echo MRI pulse sequence. Notably, such a contiguous acquisition can be combined with fully automated segmentation of adipose and lean soft tissue as well as quantification of abdominal visceral and subcutaneous depots $(15,16)$. Moreover, the ability to use higher magnetic field strength 3 Tesla (3T) scanners offers the potential for improved signal-to-noise ratio (SNR) and contrast-to-noise ratio (CNR), which can increase spatial resolution and/or reduce scan time (17).

Our overarching hypothesis is that utilization of fat-water MRI (FWMRI) to identify and quantify adipose and lean soft tissue will provide superior body composition information beyond what can be acquired from current imaging methods. As a critical first step, in this study we determined the precision of FWMRI in a manner similar to that recently reported for other novel magnetic resonance approaches (18). Thus, we rigorously compared repeated measurements of adipose and lean soft tissue in obese women acquired by FWMRI to comparable regions acquired by DEXA, the most frequently used imaging tool for assessment of body composition.

\section{Methods}

\section{Subjects}

Twelve women with BMI 30-39.9 kg/m² who were newly enrolled in a clinical trial for weight loss were recruited to have FWMRI and DEXA scans prior to initiation of a calorically restricted diet. All subjects provided written informed consent and the study was approved by the Vanderbilt University Institutional Review Board. Subjects had no history of cancer, diabetes, cardiovascular, liver or kidney disease; no weight change of greater than $2.3 \mathrm{~kg}$ within 3 months; no metal implants or other contraindications for MRI; and tested negative for pregnancy immediately prior to scanning. Acquisition of anthropometry, DEXA 
and FWMRI data from each subject was obtained at the Vanderbilt Clinical Research Center (CRC) and the Vanderbilt University Institute of Imaging Science (VUIIS) within a fourhour interval in the morning. Subjects had been instructed to avoid alcohol, excessive caffeine intake, and physical activity for 24 hours prior to study visits, and to fast from 9:00pm until arrival at 7:00am. Subjects remained in the fasted state and emptied their bladders prior to all scans.

\section{Anthropometry}

Subjects' height was obtained without shoes to the nearest $0.1 \mathrm{~cm}$ on a wall-mounted stadiometer, and weight was measured while wearing medical scrubs on a digital platform scale to the nearest $0.1 \mathrm{~kg}$. Waist and hip circumferences $( \pm 0.1 \mathrm{~cm})$ were measured by positioning a flexible measuring tape above the right iliac crest and at the full extension of the buttocks, respectively. Two derivative indices of adiposity, body mass index (BMI) from dividing weight by height squared and body adiposity index (BAI) from dividing hip circumference by height squared (19) were calculated from the anthropometric data.

\section{Dual Energy X-Ray Absorptiometry}

DEXA measurements were acquired by a certified densitometrist using a Lunar iDXA whole-body scanner (GE Healthcare, Madison WI). Analysis of DEXA images was performed using enCORE 2007 software version 11.40.004. Prior to image acquisitions, total body phantom calibration was performed according to manufacturer procedures. Subjects removed all extraneous personal items that would interfere with the accuracy of DEXA results. No subjects had body width that exceeded the dimensions of the scanner window. Scan time ranged from 6.5 - 12.5 minutes depending on DEXA-calculated abdominal sagittal thickness (20).

\section{Fat-Water Magnetic Resonance Imaging}

Subjects were scanned on a Philips Achieva 3T scanner (Philips Healthcare, Best, Netherlands) equipped with a dual gradient set capable of $40 \mathrm{mT} / \mathrm{m}$ peak strength and 200 $\mathrm{mT} / \mathrm{m} / \mathrm{ms}$ peak slew rate in the serial operation mode. A table-top extender was used to extend the stroke of the patient couch in the head-to-foot direction to enable an increased FOV length to 1.632 meters (approximately 5 feet 4 inches) in the superior-inferior direction. Subjects entered the MRI scanner feet first in the supine position with arms bent above the head using pillow and table supports. To monitor respiratory activity and breathhold compliance, an air-filled bellows was placed on the subject's abdomen with a Velcro® strap. Breath-holds were performed for 15 seconds each at table positions from the shoulders to the waist.

The integrated quadrature body coil (QBC) was used for transmit and receive of MRI sequences. The multi-station protocol included 17 table positions. Each of the 17 stacks comprised a multi-slice, multi-gradient-echo (known as multiple fast field echo or mFFE) acquisition with twelve $8 \mathrm{~mm}$ slices with slice gap set to zero. Other acquisition details included repetition time $(\mathrm{TR})=75 \mathrm{~ms}$; echo times $(\mathrm{TE})$ of TE $1 / \mathrm{TE} 2 / \mathrm{TE} 3(\mathrm{~ms})=$ $1.34 / 2.87 / 4.40$; flip angle $(\mathrm{FA})=20^{\circ}$; water fat shift $(\mathrm{WFS})=0.325$ pixels; readout sampling bandwidth $(\mathrm{BW})=1335.5 \mathrm{~Hz} /$ pixel; axial in-plane field of view $(\mathrm{FOV})=500 \mathrm{~mm}$ 
$\times 390 \mathrm{~mm}$; acquired in-plane matrix size $=252 \times 195$; and acquired voxel size $=2 \mathrm{~mm} \times 2$ $\mathrm{mm} \times 8 \mathrm{~mm}$. Automated linear shimming was performed for each slice stack. Flyback gradients were employed between echoes so that the chemical shift direction for all echo readouts was the same. Data acquisition time averaged 4 minutes and 16 seconds with approximately 5 additional minutes for table movement, preparation phases at each table position, and breath-holding pauses. Subjects' total time in the scanner including subject positioning, scout imaging and image planning was 20-25 minutes.

\section{Regions of Interest}

From DEXA and FWMRI acquisitions, we report four tissue compartments: gross body adipose tissue (GBAT), total trunk adipose tissue (TTAT), gross body lean soft tissue (TLST) and total trunk lean soft tissue (TTLT). With FWMRI, GBAT and GBLST included all tissue visible in the scanner FOV. We also report SAT and VAT from FWMRI. We manually customized the DEXA trunk region of interest (ROI) to match the upper and lower anatomical boundaries of the visceral region delineated by the automated MR image segmentation algorithm, which defined the abdominal subvolume as extending from the base of the lungs to the femoral heads. Figure 1 displays the coronal DEXA image (on the left) and $1^{\text {st }}$ echo maximum intensity projection from the FWMRI acquisition (on the right) for subject \#1. The DEXA ROI in the head-to-foot direction was manually set to match the abdominal subvolume identified by the FWMRI segmentation algorithm by placing a rostral horizontal line at the lower base of the lung and a caudal horizontal line at the top of the femoral head. The left-to-right boundaries of the ROI were adjusted to exclude as much of the arms as possible.

\section{Fat-Water Magnetic Resonance Imaging Processing}

Complex (real and imaginary) images for each of the acquired three echo times were reconstructed and exported in Digital Imaging and Communications in Medicine (DICOM) standard format for off-line processing. Separation of water and fat signal requires knowledge of the main magnetic field map, and essentially all water/fat separation algorithms rely on an assumption of spatial smoothness of the main magnetic field. Water and fat images were reconstructed using a recently published algorithm by Berglund et al. (21) in which fat and water signal components were found by least squares fitting (22) after resolving the magnetic field map using a 3D multiple seed-point region growing algorithm with a dynamic path sensitive to field inhomogeneity, solving regions of homogeneous magnetic field before inhomogeneous or noisy regions. Berglund's 3-point Dixon algorithm, which directly finds two analytical solutions for the background static field phasor term, is valid as long as the echo spacing between the 1st and 2nd echoes matches the spacing between the 2nd and 3rd echo. When applied to whole-body 3D data sets acquired at $1.5 \mathrm{~T}$, the method was found by two blinded radiologists to have the best image quality (in terms of fat water "swaps") compared to two reference methods: an advanced version of the "iterative decomposition of water and fat with echo asymmetric and least-squares estimation" IDEAL approach (23) as well as a multi-resolution golden section search (MRGS) method (24). The employed fat-water separation algorithm used a multi-peak model with nine peaks set with fixed spectral locations and relative amplitudes. Values of the chemical shifts and of the relative amplitudes were taken from a liver spectroscopy study 
of 121 human subjects (25). The reconstructed fat and water image volumes were input into a fully automated segmentation algorithm (16) that first localized the abdominal subvolume and then separated VAT from SAT within the identified abdominal subvolume. Lean soft tissue was quantified by measuring the total volume of the voxels with fat signal fractions below 50\%. Adipose and lean soft tissue volumes were multiplied by published density values of $0.923 \mathrm{~kg} / \mathrm{L}$ and $1.05 \mathrm{~kg} / \mathrm{L}$, respectively, to convert volume to mass $(\mathrm{kg})(13,26)$. Typical post-processing times for the fat-water separation and automated segmentation algorithms were two and three minutes respectively for the size of image files in this study. Figure 2 presents a single coronal slice (top left), single sagittal slice (top middle), a montage of 20 axial slices (top right), and a histogram of fat signal percentage for all voxels in the 3D MRI fat volume of subject \#5. As seen in these images, VAT is displayed in red. Across the axial slices, the identified VAT voxels are spatially consistent and are not contaminated by SAT or other voxels. Importantly, the algorithm is designed to automatically exclude bone marrow and intramuscular adipose tissue in the area of the spine, which would confound adipose tissue measures. On the scale of fat signal percentage in the displayed histogram, two distinct populations of voxels are discernable. The populations are widely separated with long tails. Applying the well-known Otsu method (27) to determine an optimal threshold that simultaneously minimizes intraclass variance and maximizes interclass variance yields an optimal threshold of $49.8 \%$ for this example data to distinguish the fatty and lean voxels which is very close to the employed threshold of $50 \%$.

\section{Statistical Analysis}

Statistical analyses were conducted using SPSS version 19.0 (IBM Corp., Armonk, NY) and MATLAB version 7.10.0.499 R2010a (MathWorks Inc., Natick, MA) with the level of significance set at $\alpha=0.05$. Data are presented as mean \pm standard deviation (sd). As values were not normally distributed, Wilcoxon signed-rank tests were performed to assess the significance of the observed differences between DEXA and FWMRI for TAT, TTAT, TLST, and TTLST. Percent differences were calculated as (FWMRI - DEXA)/DEXA $\times$ $100 \%$.

\section{Coefficient of Variation}

To determine the precision of DEXA and FWMRI methods, we performed test-retest measurements and then calculated coefficients of variation (CV) for the tissue masses estimated by each modality. CV was determined from the square root of the mean withingroup variance of a one-way ANOVA (with each subject as a group) divided by the grand mean (28), and multiplied by 100 to express CV as a percentage. Thereafter, all analyses were performed using the averaged value of the test-retest data. As complete retest FWMRI scans were not available for subjects \#5 and \#7, FWMRI values for these subjects represent one measurement only and they were excluded in CV analysis.

\section{Correlation Coefficients}

The concordance correlation coefficient $\left(\rho_{\mathrm{c}}\right)$, often used in comparing quantitative methods, measures accuracy by determining how much the linear relationship between two variables deviates from the line of concordance and precision by how far each observation deviates 
from the fitted line (29). The more commonly known Pearson correlation coefficient $\left(\rho_{r}\right)$ is related to $\rho_{\mathrm{c}}$ via the bias correction factor $\left(\mathrm{C}_{\mathrm{b}}\right)$, which is a ratio of the two coefficients $\left(\rho_{\mathrm{c}} / \rho_{\mathrm{r}}\right)$ (30). The value of $C_{b}$ is within the range of 0 to 1 , and thus, measures how far the best-fit line deviates from the $45^{\circ}$ line (accuracy). Only using the Pearson correlation coefficient would measure how far each observation deviates from the best-fit line (precision) but not accuracy. Here we report $\rho_{\mathrm{c}}$, bias correction factors and asymptotic $95 \%$ confidence intervals for $\rho_{\mathrm{c}}$. Spearman's Rho correlation coefficients $\left(\rho_{\mathrm{s}}\right)$ were also calculated to identify any monotonic relationships between adipose and lean soft tissue discrepancies (difference between DEXA and FWMRI) with anthropometric measures.

\section{Bland-Altman Analysis}

The Bland-Altman method (31) was used to assess the limits of agreement (mean difference $\pm 1.96 \mathrm{sd}$ of the difference) between DEXA and FWMRI measures. As such the difference between the methods ( $\mathrm{y}$-axis) was plotted against the mean of the methods (x-axis). Linear dependence trends in the Bland-Altman plots were calculated using a least squares fit to a first order polynomial. The coefficient of determination $\left(\mathrm{r}^{2}\right)$ and significance level of each linear fit were also calculated.

\section{Results}

Descriptive characteristics of the twelve female subjects are presented in Table 1. Five of the women self-identified as Caucasian and seven as African American, but no significant differences were detected by ethnicity. Subjects had an average BMI of $34.3 \pm 2.8 \mathrm{~kg} / \mathrm{m}^{2}$ and an average BAI $39.5 \pm 4.8 \%$ indicating Class I obesity.

Average adipose and lean soft tissue masses measured by DEXA and FWMRI are presented in Table 2 for each subject. For adipose tissue, mean GBAT was $39.9 \pm 7.5 \mathrm{~kg}$ by FWMRI and $39.8 \pm 6.9 \mathrm{~kg}$ by DEXA, and mean TTAT was $14.0 \pm 3.4 \mathrm{~kg}$ by FWMRI and $15.7 \pm 3.5$ $\mathrm{kg}$ by DEXA. The mean FWMRI measurements for SAT and VAT were $11.6 \pm 2.6 \mathrm{~kg}$ and $2.1 \pm 1.0 \mathrm{~kg}$, respectively. For lean soft tissue, mean GBLST was $39.8 \pm 5.4 \mathrm{~kg}$ by FWMRI and $45.8 \pm 7.3 \mathrm{~kg}$ by DEXA, and mean TTLST was $11.9 \pm 1.7 \mathrm{~kg}$ by FWMRI and $15.1 \pm 2.7$ $\mathrm{kg}$ by DEXA.

\section{Coefficient of Variation}

CVs for GBAT, TTAT, GBLST and TTLST are presented and show that reproducibility measurements were $<3 \%$ for DEXA and FWMRI (Table 2). For DEXA, CVs were $0.58 \%$, $1.35 \%, 0.42 \%$ and $1.02 \%$, for GBAT, TTAT, GBLST and TTLST, respectively. For FWMRI, CVs were $0.80 \%, 2.08 \%, 0.60 \%$ and $2.43 \%$, respectively. The CVs for FWMRI quantification of SAT and VAT were $2.11 \%$ and $2.62 \%$, respectively.

\section{Comparison of Tissue Masses}

Concordance correlation coefficients, coefficients of bias, and mean differences between FWMRI and DEXA are presented in Table 3. None of the 95\% confidence intervals include zero, indicating statistically significant correlations between FWMRI and DEXA for GBAT, TTAT, GBLST and TTLST. The strength of the concordance correlation was highest for 
GBAT with $\rho_{\mathrm{c}}=0.978(0.923,0.994)$ and $\mathrm{C}_{\mathrm{b}}=0.996\left(\rho_{\mathrm{r}}=0.982, p<0.0001\right)$. A Wilcoxon signed-rank test also showed no significant difference $(0.1 \pm 1.5 \mathrm{~kg}, p=0.75)$ between FWMRI and DEXA measures for GBAT. However, concordance correlations for TTAT, GBLST and TTLST showed negative biases toward FWMRI. Correlation between FWMRI and DEXA for TTAT was $\rho_{\mathrm{c}}=0.802(0.468,0.935)$ and $\mathrm{C}_{\mathrm{b}}=0.884\left(\rho_{\mathrm{r}}=0.907, p<0.0001\right)$, but a Wilcoxon signed-rank test revealed a significant bias of $-1.7 \pm 1.5 \mathrm{~kg}(-10.4 \pm 9.1 \%, p$ $=0.008)$.

Concordance correlation of GBLST was $\rho_{\mathrm{c}}=0.629(0.324,0.816)$ and $\mathrm{C}_{\mathrm{b}}=0.646\left(\rho_{\mathrm{r}}=0.974, p\right.$ $<0.0001)$, and a Wilcoxon signed-rank test showed a significant bias of $-6.0 \pm 2.4 \mathrm{~kg}$ $(-12.8 \pm 3.8 \%, p=0.003)$. Concordance correlation of TTLST was the lowest of the compared tissue masses with $\rho_{\mathrm{c}}=0.400(0.120,0.620)$ and $\mathrm{C}_{\mathrm{b}}=0.423\left(\rho_{\mathrm{r}}=0.944, p<0.0001\right)$. A Wilcoxon signed-rank test confirmed a significant bias of $-3.2 \pm 1.2 \mathrm{~kg}(-20.9 \pm 5.2 \%, p$ $=0.003)$.

\section{Bland-Altman Analysis}

Bland-Altman plots for GBAT and TTAT (Figure 3) show good agreement between FWMRI and DEXA with the limits of agreement for GBAT from $-2.8 \mathrm{~kg}$ to $+3.1 \mathrm{~kg}$ and for TTAT from $-4.6 \mathrm{~kg}$ to $+1.2 \mathrm{~kg}$. These ranges include zero and linear regression of the method difference against method average showed no statistically significant trend with $\mathrm{r}^{2}=$ $0.17(p=0.18)$ for GBAT and $\mathrm{r}^{2}=0.00(p=0.92)$ for TTAT.

Bland-Altman plots for GBLST and TTLST (Figure 4) indicate there is less agreement between FWMRI and DEXA for lean soft tissue than for adipose tissue. Neither the limits of agreement for GBLST $(-10.8 \mathrm{~kg}$ to $-1.3 \mathrm{~kg}$ ) or TTLST $(-5.6 \mathrm{~kg}$ to $-0.9 \mathrm{~kg})$ include zero. The lean soft tissue plots reveal an increasing negative bias in the FWMRI minus DEXA difference with increasing lean soft tissue mass. Linear regression of the method difference against method average confirmed that this trend was statistically significant for GBLST $\left(r^{2}\right.$ $=0.66, p=0.0013)$ and TTLST $\left(r^{2}=0.67, p=0.0011\right)$.

\section{FWMRI versus DEXA Differences}

No anthropometric measures or derived values (BMI and BAI) were significantly correlated with the difference between FWMRI and DEXA for TTAT. Waist circumference, hip circumference and BMI were also not significantly correlated with the differences observed in GBLST or TTLST. However, as presented in Figure 5, the differences between FWMRI and DEXA for GBLST and TTLST were significantly correlated with subject height $\left(\rho_{\mathrm{s}}=\right.$ -0.811 and $\left.\rho_{\mathrm{s}}=-0.790\right)$, weight $\left(\rho_{\mathrm{s}}=-0.895\right.$ and $\left.\rho_{\mathrm{s}}=-0.832\right)$ and abdominal sagittal thickness $\left(\rho_{\mathrm{s}}=-0.804\right.$ and $\left.\rho_{\mathrm{s}}=-0.601\right)$. Additionally, BAI was correlated with TTLST difference $\left(\rho_{\mathrm{s}}=0.608, p=0.004\right)$.

\section{Discussion}

The key finding from this study is demonstration that the precision of the implemented contiguous 3D fat-water MR imaging technique at 3 Tesla makes it a compelling alternative to DEXA for quantifying body composition in obesity primarily because this method offers the important advantage of quantifying VAT and SAT. As recent MR imaging body 
composition studies have reported findings from adipose tissue mass only $(16,32)$, the present study further extends the evidence by presenting data on lean soft tissue mass which is an important determinant of insulin sensitivity and has a critical role in the pathophysiology of many disease processes including obesity-associated sarcopenia and cachectic syndromes.

The coefficient of variation for repeated measurements using FWMRI in obese women was less than $1 \%$ for GBAT and GBLST. Confirming the excellent precision of the FWMRI method, the CVs for GBAT and GBLST were comparable to those of DEXA (less than $0.6 \%$ ). Additionally, FWMRI CVs for trunk adipose tissue (TTAT) and trunk lean soft tissue (TTLST) were less than 2.5\%, and CVs for SAT and VAT were less than 2.7\%. Our findings demonstrate greater precision for FWMRI at 3T than those previously reported at 1.5T where GBAT CV was $2.32 \%$ and TTAT CV was $2.25 \%$ (16). We observed slightly less precision in the CVs for FWMRI trunk tissues compared to DEXA where TTAT and TTLST CVs were less than $1.4 \%$. We found no published reports comparing the reproducibility of FWMRI for GBLST or TTLST.

Consistent with the overall precision, we found that FWMRI measures of GBAT in these obese women correlated strongly with DEXA $\left(\rho_{\mathrm{c}}=0.978\right)$. However, it appeared that FWMRI underestimated TTAT. Kullberg et al (32) have previously reported that FWMRI at 1.5T underestimated VAT when compared to computed tomography and suggest that abdominal soft tissue motion and partial volume effects may contribute to VAT signal loss. Similar effects may explain the lower TTAT measured by FWMRI in this study. Partial volume effects are a potential confounding factor in general for the thresholded adipose tissue segmentation approach applied in this study while being less of a concern for fat signal fraction integration approaches. However, a number of known confounding factors (RF transmit and receive inhomogeneity, $\mathrm{T} 1$ relaxation, $\mathrm{T} 2 *$ relaxation, eddy currents, noise bias, multiple fat resonances) must be addressed for the signal fat fraction to accurately reflect the true proton density fat fraction. Another confounding factor arises from intramuscular adipose tissue included in the VAT volume. The automated segmentation algorithm (16) excludes bone marrow and intramuscular adipose tissue in the abdominal subvolume in the region of the spine and back. However, the exclusion of intramuscular tissue throughout the abdomen is not perfect, as is visible in some axial sections displayed in Figure 2, and is a focus of ongoing development.

Though correlations between FWMRI and DEXA estimates of lean soft tissue were strong for GBLST and TTLST regions, our findings indicate a significant difference between the two methods that increased in magnitude with taller, heavier and thicker subjects. As the women in the present study ranged in height from 149.9 to $177.8 \mathrm{~cm}$, some extremity tissue was excluded from the FWMRI field of view, which was limited to a maximum of 163.2 $\mathrm{cm}$.. Thus, the discrepancy in GBLST is accounted for by missing the most distal portions of the head, feet, hands and forearms (which were bent directly overhead) We recognize that tissue not captured within the FWMRI FOV is a primary source of error in the FWMRI quantification. However, future studies can address this shortcoming by matching overhead arm position for DEXA and FWMRI prior to comparison and by software modifications to increase scanning coverage in the head-to-foot direction. Another limitation of the current 
quantification method is the use of a binary, all-or-none, classification of FWMRI voxels as either water (lean soft tissue) or fat (adipose tissue). Once confounding factors such as transmit and receive RF field inhomogeneity and $\mathrm{T} 1$ bias are better-compensated, more accurate estimates of fat volume (and mass) could be obtained using the fractional fat signal percentage in each FWMRI voxel.

However, incomplete coverage of the extremities does not explain the difference in TTLST observed between FWMRI and DEXA because the entire trunk region was well sampled in the FWMRI FOV. The bias between FWMRI and DEXA in TTLST, may be explained by overestimation of lean soft tissue by DEXA. Such overestimation has been observed in several prior studies and attributed to assumptions in DEXA algorithms for estimating appendicular skeletal muscle $(8,9)$. It must also be recognized that direct comparison of soft tissue measured by FWMRI and DEXA methods is limited by the fact that FWMRI measures tissue volume, which must be converted to mass by using assumed densities for soft tissue (33). Unfortunately, comparison between FWMRI and CT was contraindicated in the present study because of the need for performing repeated measures to determine repeatability and the substantial concern with CT-associated radiation exposure.

Although MRI scanning time has been perceived to be an impediment to widespread use of MRI in the past, the FWMRI scan time in this study was comparable to that of DEXA acquisition. Other faster variations of multi-echo DIXON methods different from the threeecho method employed in this study are available. For example, a two-echo method has the advantage of shorter acquisition time, but acquiring three echoes improves identification of the water and fat components, which simplifies signal separation. Acquiring more than three echoes enables additional unknowns such as $\mathrm{T} 2 *$ to be numerically fit, but the acquisition of more echoes increases scan time or requires a reduction in spatial resolution. The FWMRI scan time can be further accelerated using parallel imaging methods such as sensitivity encoding (SENSE) which requires an array of multiple receive-only radio frequency (RF) surface coils (34). Scan time could also be reduced with a continuously moving table strategy (16) or by using a data sampling acceleration strategy combined with an advanced image reconstruction approach such as compressive sampling (35).

In the present study, the absence of surface RF coils maximized subject comfort, compatibility with larger subjects, and simplified the multiple table-station scanning approach. Importantly, the current speed of FWMRI acquisition makes it as affordable as DEXA in research and clinical settings. For example, at our institution the charge for the 30minute time slot required to complete a FWMRI scan on the research 3T MR scanner is equivalent to the charge for a 30-minute DEXA time slot. It is also noteworthy that FWMRI in the present study was performed on a clinical scanner, which clearly increases the potential for widespread utilization of this method. Yet, we acknowledge that MRI scanning at $3 \mathrm{~T}$ presents increased challenges related to static magnetic field (B0) and RF transmit field (B1) inhomogeneity (17) which can cause geometric distortion, signal loss and signal intensity inhomogeneity that can confound accurate fat and water separation as well as increase error in volume measurements. In cases where static magnetic field (B0) homogeneity becomes problematic, alternative shimming strategies such as slice-wise 
dynamic shimming can be pursued (36). Challenges related to RF transmit field (B1) inhomogeneity that may arise could be addressed by a parallel RF transmit 3T scanner (37).

In conclusion, we have demonstrated that gross body FWMRI imaging performed at $3 \mathrm{~T}$ is rapid, precise, repeatable and potentially cost effective. In the obese women studied, FWMRI had excellent concordance with DEXA for the measurement of gross body adipose tissue. Moreover, because FWMRI data is three-dimensional and reliably divides the MR signal into separate fat and water components, this method is able to go advance the study of body composition and distinguish adipose tissue topography to quantify visceral and subcutaneous abdominal tissue depots. While recent reports on quantifying body composition using magnetic resonance spectroscopy (quantitative magnetic resonance) also show great promise with regard to sensitivity and specificity for certain applications (18, 38), such spectroscopy-based approaches provide little or no information about the spatial distribution of adipose and lean soft tissue. In addition, the chemical-shift-based separation of fat and water signals employed in the FWMRI approach described is more advanced than MRI fat quantification approaches that are based on standard relaxation contrast mechanisms, which are not amenable to automated segmentation and quantification algorithms (39). Importantly, future applications of the FWMRI method described will also allow quantification of ectopic fat (ie, intermuscular adipose tissue and lipid burden within organs such as the liver, pancreas and heart) (40). Finally, when compared to DEXA and other non-MR methods (ie, computed tomography), FWMRI is prudent because it requires no exposure to ionizing radiation. Thus, it can be used safely in children and in longitudinal studies that require serial measurements to capture changes in body composition. Hence, the implementation of a 3T multi-station multi-gradient-echo based fat-water imaging method described in this work combined with a fully-automated post-processing algorithm is currently being applied to investigate physiological and metabolic mechanisms that lead to the development of obesity and its metabolic consequences.

\section{Supplementary Material}

Refer to Web version on PubMed Central for supplementary material.

\section{Acknowledgments}

The authors thank Taren B. Spence, RD for study data collection, Charles DeMarcus Keil for data entry, and VUIIS MR technologists, Robin Avison and Donna Butler, for MR scanning support. We also thank the study participants for their time and commitment. Study concept and design: HJS, KDN, MJA, JK and EBW. Study protocol and data collection: HJS and EBW. Data analysis: HJS, EBW, JK, and JB. Interpretation of data: HJS, KDN, MJA, JK, EBW. Manuscript writing and review of intellectual content: HJS, KDN, MJA, LJ, JK, JB, MB, and EBW. This work was supported by The Dr. Robert C. and Veronica Atkins Foundation (HJS), the Vanderbilt CTSA grant 1 UL1 RR024975 from the National Center for Research Resources, National Institutes of Health (HJS), the Tennessee Valley Healthcare System, NIH grants DK064857 and DK069927, and the Vanderbilt Diabetes Research and Training Center grant DK020593 (KDN).

This study was funded by The Dr. Robert C. and Veronica Atkins Foundation and the Vanderbilt CTSA grant 1 UL1 RR024975 from the National Center for Research Resources, National Institutes of Health. The study was also supported in part by the Tennessee Valley Healthcare System, NIH grants DK064857 and DK069927, and the Vanderbilt Diabetes Research and Training Center grant DK020593. Lars Johansson is partly employed by AstraZeneca. Morten Bruvold is employed by Philips Healthcare. 


\section{References}

1. Bouchard C, Despres JP, Mauriege P. Genetic and nongenetic determinants of regional fat distribution. Endocr Rev. 1993 Feb; 14(1):72-93. [PubMed: 8491156]

2. Smith SR, Lovejoy JC, Greenway F, Ryan D, deJonge L, de la Bretonne J, et al. Contributions of total body fat, abdominal subcutaneous adipose tissue compartments, and visceral adipose tissue to the metabolic complications of obesity. Metabolism. 2001 Apr; 50(4):425-35. [PubMed: 11288037]

3. Nedungadi TP, Clegg DJ. Sexual dimorphism in body fat distribution and risk for cardiovascular diseases. J Cardiovasc Transl Res. 2009 Sep; 2(3):321-7. [PubMed: 20560019]

4. Ross R. Advances in the application of imaging methods in applied and clinical physiology. Acta Diabetol. 2003 Oct; 40(Suppl 1):S45-50. [PubMed: 14618432]

5. Scherzer R, Shen W, Bacchetti P, Kotler D, Lewis CE, Shlipak MG, et al. Comparison of dualenergy X-ray absorptiometry and magnetic resonance imaging-measured adipose tissue depots in HIV-infected and control subjects. Am J Clin Nutr. 2008 Oct; 88(4):1088-96. [PubMed: 18842798]

6. Sierra-Johnson J, Johnson BD, Bailey KR, Turner ST. Relationships between insulin sensitivity and measures of body fat in asymptomatic men and women. Obes Res. 2004 Dec; 12(12):2070-7. [PubMed: 15687409]

7. Kamel EG, McNeill G, Han TS, Smith FW, Avenell A, Davidson L, et al. Measurement of abdominal fat by magnetic resonance imaging, dual-energy X-ray absorptiometry and anthropometry in non-obese men and women. Int J Obes Relat Metab Disord. 1999 Jul; 23(7):68692. [PubMed: 10454101]

8. Wang ZM, Visser M, Ma R, Baumgartner RN, Kotler D, Gallagher D, et al. Skeletal muscle mass: evaluation of neutron activation and dual-energy X-ray absorptiometry methods. J Appl Physiol. 1996 Mar; 80(3):824-31. [PubMed: 8964743]

9. Thomsen TK, Jensen VJ, Henriksen MG. In vivo measurement of human body composition by dualenergy X-ray absorptiometry (DXA). Eur J Surg. 1998 Feb; 164(2):133-7. [PubMed: 9537721]

10. Thomas EL, Saeed N, Hajnal JV, Brynes A, Goldstone AP, Frost G, et al. Magnetic resonance imaging of total body fat. J Appl Physiol. 1998 Nov; 85(5):1778-85. [PubMed: 9804581]

11. Börnert P, Keupp J, Eggers H, Aldefeld B. Whole-body 3D water/fat resolved continuously moving table imaging. J Magn Reson Imaging. 2007 Mar; 25(3):660-5. [PubMed: 17326078]

12. Machann J, Thamer C, Schnoedt B, Haap M, Haring HU, Claussen CD, et al. Standardized assessment of whole body adipose tissue topography by MRI. J Magn Reson Imaging. 2005 Apr; 21(4):455-62. [PubMed: 15778954]

13. Abate N, Burns D, Peshock RM, Garg A, Grundy SM. Estimation of adipose tissue mass by magnetic resonance imaging: validation against dissection in human cadavers. J Lipid Res. 1994 Aug; 35(8):1490-6. [PubMed: 7989873]

14. Ross R, Leger L, Morris D, de Guise J, Guardo R. Quantification of adipose tissue by MRI: relationship with anthropometric variables. J Appl Physiol. 1992 Feb; 72(2):787-95. [PubMed: 1559959]

15. Sjostrom L, Kvist H, Cederblad A, Tylen U. Determination of total adipose tissue and body fat in women by computed tomography, 40K, and tritium. Am J Physiol. 1986 Jun; 250(6 Pt 1):E73645. [PubMed: 3717334]

16. Kullberg J, Johansson L, Ahlstrom H, Courivaud F, Koken P, Eggers H, et al. Automated assessment of whole-body adipose tissue depots from continuously moving bed MRI: a feasibility study. J Magn Reson Imaging. 2009 Jul; 30(1):185-93. [PubMed: 19557740]

17. Soher BJ, Dale BM, Merkle EM. A review of MR physics: 3T versus 1.5T. Magn Reson Imaging Clin N Am. 2007 Aug; 15(3):277-90. v. [PubMed: 17893049]

18. Gallagher D, Thornton JC, He Q, Wang J, Yu W, Bradstreet TE, et al. Quantitative magnetic resonance fat measurements in humans correlate with established methods but are biased. Obesity (Silver Spring). 2010 Oct; 18(10):2047-54. [PubMed: 20448539]

19. Bergman RN, Stefanovski D, Buchanan TA, Sumner AE, Reynolds JC, Sebring NG, et al. A Better Index of Body Adiposity. Obesity (Silver Spring). 2011 Mar 3.

20. Jebb SA, Goldberg GR, Elia M. DXA measurements of fat and bone mineral density in relation to depth and adiposity. Basic Life Sci. 1993; 60:115-9. [PubMed: 8110089] 
21. Berglund J, Johansson L, Ahlstrom H, Kullberg J. Three-point Dixon method enables whole-body water and fat imaging of obese subjects. Magn Reson Med. 2010 Jun; 63(6):1659-68. [PubMed: 20512869]

22. An L, Xiang QS. Chemical shift imaging with spectrum modeling. Magn Reson Med. 2001 Jul; 46(1):126-30. [PubMed: 11443718]

23. Yu H, Reeder SB, Shimakawa A, Brittain JH, Pelc NJ. Field map estimation with a region growing scheme for iterative 3-point water-fat decomposition. Magn Reson Med. 2005 Oct; 54(4):1032-9. [PubMed: 16142718]

24. Lu W, Hargreaves BA. Multiresolution field map estimation using golden section search for waterfat separation. Magn Reson Med. 2008 Jul; 60(1):236-44. [PubMed: 18581397]

25. Hamilton G, Yokoo T, Bydder M, Cruite I, Schroeder ME, Sirlin CB, et al. In vivo characterization of the liver fat (1)H MR spectrum. NMR Biomed. 2011 Aug; 24(7):784-90. [PubMed: 21834002]

26. Chowdhury B, Sjostrom L, Alpsten M, Kostanty J, Kvist H, Lofgren R. A multicompartment body composition technique based on computerized tomography. Int J Obes Relat Metab Disord. 1994 Apr; 18(4):219-34. [PubMed: 8044196]

27. Otsu N. Threshold Selection Method from Gray-Level Histograms. Ieee T Syst Man Cyb. 1979; 9(1):62-6.

28. Bland, M. An introduction to medical statistics. 2. Oxford; New York: Oxford University Press; 1995.

29. Lin LI. A concordance correlation coefficient to evaluate reproducibility. Biometrics. 1989 Mar; 45(1):255-68. [PubMed: 2720055]

30. Crawford SB, Kosinski AS, Lin HM, Williamson JM, Barnhart HX. Computer programs for the concordance correlation coefficient. Comput Methods Programs Biomed. 2007 Oct; 88(1):62-74. [PubMed: 17709153]

31. Bland JM, Altman DG. Statistical methods for assessing agreement between two methods of clinical measurement. Lancet. 1986 Feb 8; 1(8476):307-10. [PubMed: 2868172]

32. Kullberg J, Brandberg J, Angelhed JE, Frimmel H, Bergelin E, Strid L, et al. Whole-body adipose tissue analysis: comparison of MRI, CT and dual energy X-ray absorptiometry. Br J Radiol. 2009 Feb; 82(974):123-30. [PubMed: 19168691]

33. Wang ZM, Pierson RN Jr, Heymsfield SB. The five-level model: a new approach to organizing body-composition research. Am J Clin Nutr. 1992 Jul; 56(1):19-28. [PubMed: 1609756]

34. Pruessmann KP, Weiger M, Scheidegger MB, Boesiger P. SENSE: sensitivity encoding for fast MRI. Magn Reson Med. 1999 Nov; 42(5):952-62. [PubMed: 10542355]

35. Doneva M, Bornert P, Eggers H, Mertins A, Pauly J, Lustig M. Compressed sensing for chemical shift-based water-fat separation. Magn Reson Med. 2010 Dec; 64(6):1749-59. [PubMed: 20859998]

36. Morrell G, Spielman D. Dynamic shimming for multi-slice magnetic resonance imaging. Magn Reson Med. 1997 Sep; 38(3):477-83. [PubMed: 9339449]

37. Katscher U, Bornert P. Parallel RF transmission in MRI. NMR Biomed. 2006 May; 19(3):393400. [PubMed: 16705630]

38. Swe Myint K, Napolitano A, Miller SR, Murgatroyd PR, Elkhawad M, Nunez DJ, et al. Quantitative magnetic resonance (QMR) for longitudinal evaluation of body composition changes with two dietary regimens. Obesity (Silver Spring). 2010 Feb; 18(2):391-6. [PubMed: 19696753]

39. Machann J, Thamer C, Stefan N, Schwenzer NF, Kantartzis K, Haring HU, et al. Follow-up wholebody assessment of adipose tissue compartments during a lifestyle intervention in a large cohort at increased risk for type 2 diabetes. Radiology. 2010 Nov; 257(2):353-63. [PubMed: 20713612]

40. Silver HJ, Welch EB, Avison MJ, Niswender KD. Imaging body composition in obesity and weight loss: challenges and opportunities. Diabetes Metab Syndr Obes. 2010; 3:337-47. [PubMed: 21437103] 


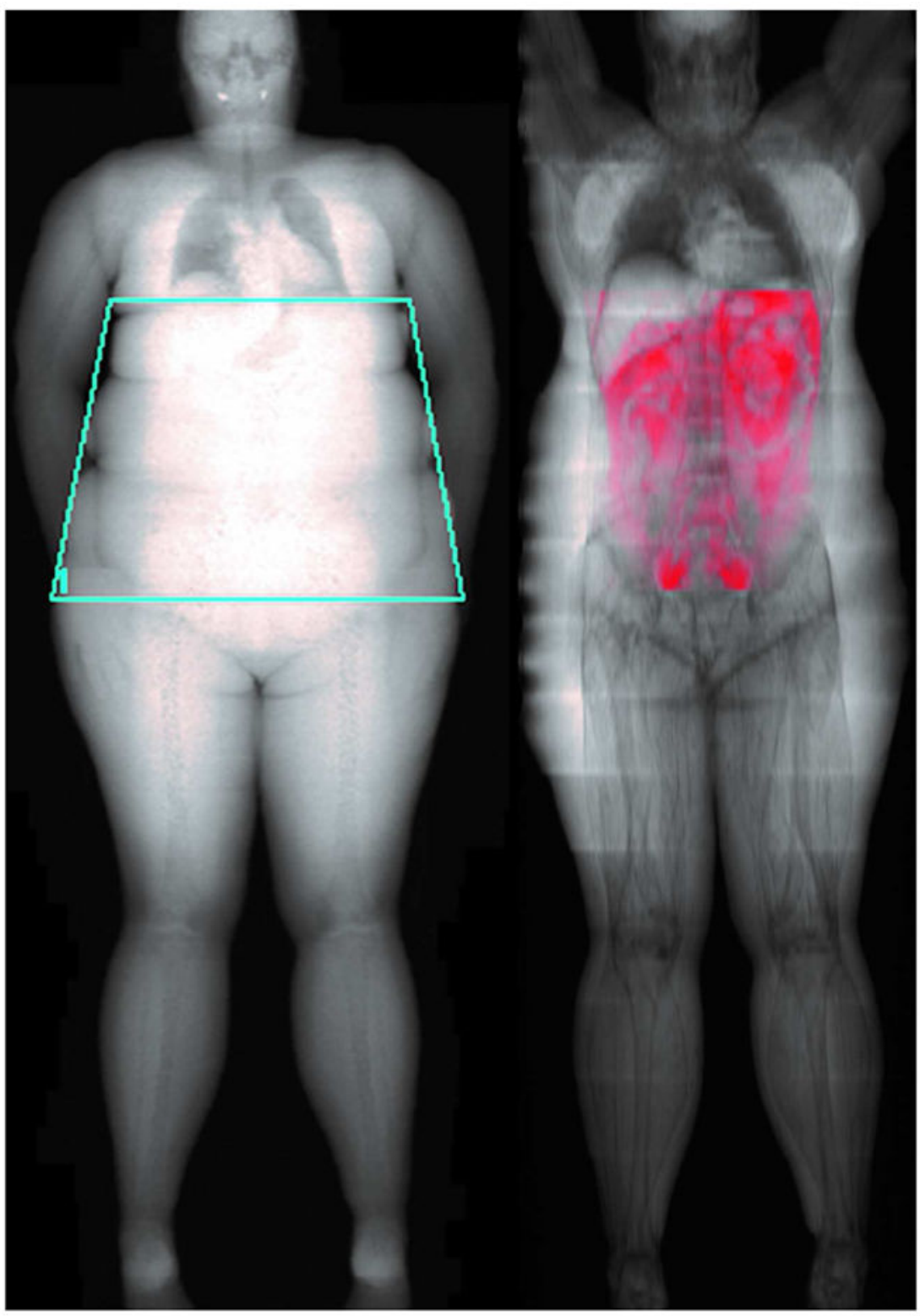

Figure 1.

Coronal DEXA image (left) and $1^{\text {st }}$ echo maximum intensity projection from the FWMRI acquisition (right) for subject \#1. The blue trapezoidal region of interest on the DEXA image was drawn to exclude as much of the arms as possible and is used for reporting the DEXA trunk (abdominal) adipose and lean soft tissue masses to compare to the FWMRI trunk measurements. The identified VAT output from the automated FWMRI segmentation algorithm is displayed in red. 

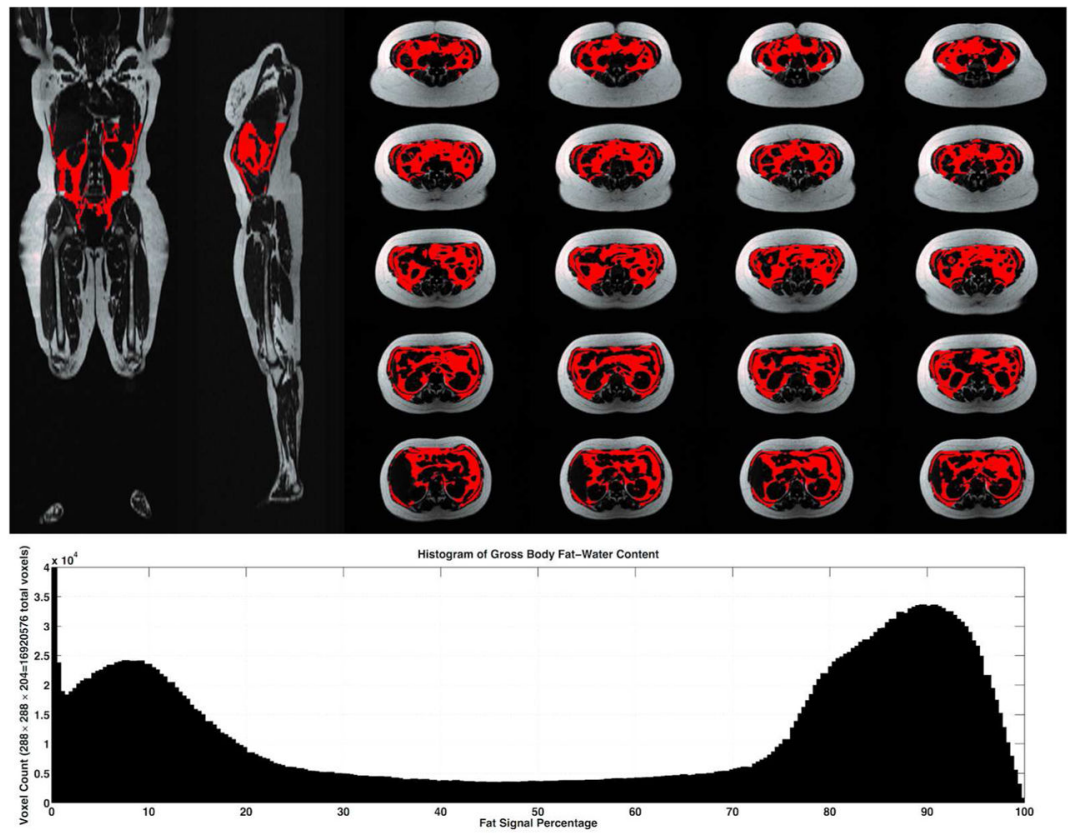

Figure 2.

Coronal cross section (top left), sagittal cross section (top middle) and axial 20-slice montage (top right) from the FWMRI acquisition showing the visceral adipose tissue segmentation (red) superimposed on the fat image for subject \#5. The images show how the VAT segmentation algorithm successfully excludes subcutaneous fat as well as the bone marrow and intramuscular adipose tissue around the spine. The histogram of fat signal percentage in the entire 3D image volume (bottom) shows two distinct populations of fatty and lean soft tissue. For the automated segmentation algorithm, any voxel with a fat signal percentage greater than $50 \%$ was considered to be fat. After excluding background voxels, applying Otsu's method (27) yields an optimal threshold level of $49.8 \%$ to separate the two voxel populations. 

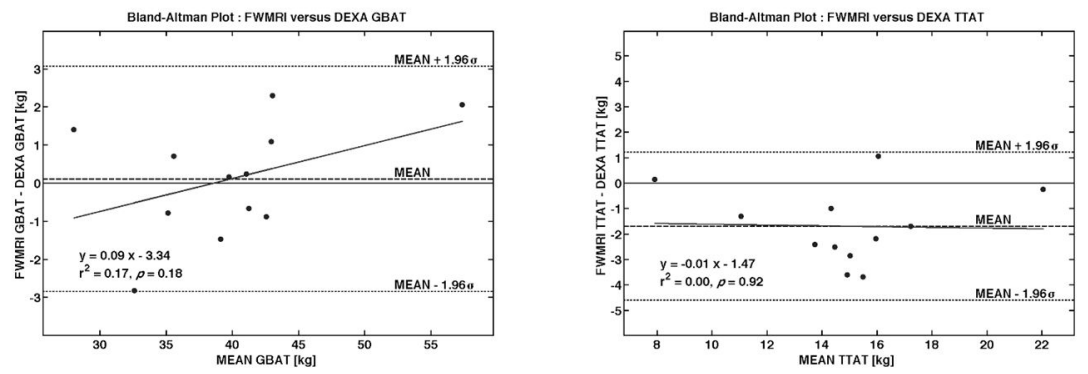

Figure 3.

Bland-Altman plots and fitted linear trends of mean versus difference between FWMRI and DEXA measurements for GBAT (left) and TTAT (right). The GBAT difference shows negligible bias and an insignificant linear trend. The agreement for total trunk adipose tissue demonstrates a small negative bias toward FWMRI underestimation. However, the zero difference line for TTAT is still within the displayed $95 \%$ confidence interval boundaries, and the TTAT differences show no significant linear trend. 

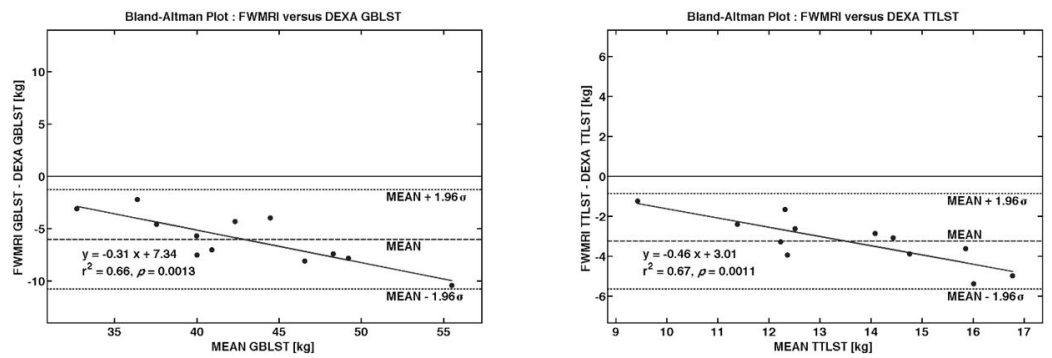

Figure 4.

Bland-Altman plots and fitted linear trends of mean versus difference between FWMRI and DEXA measurements for GBLST (left) and TTLST (right). Differences between the methods reveal a significant negative bias for FWMRI and a significant linear trend for GBLST and TTLST. Thus, the discordance increases as GBLST and TTLST increases. 

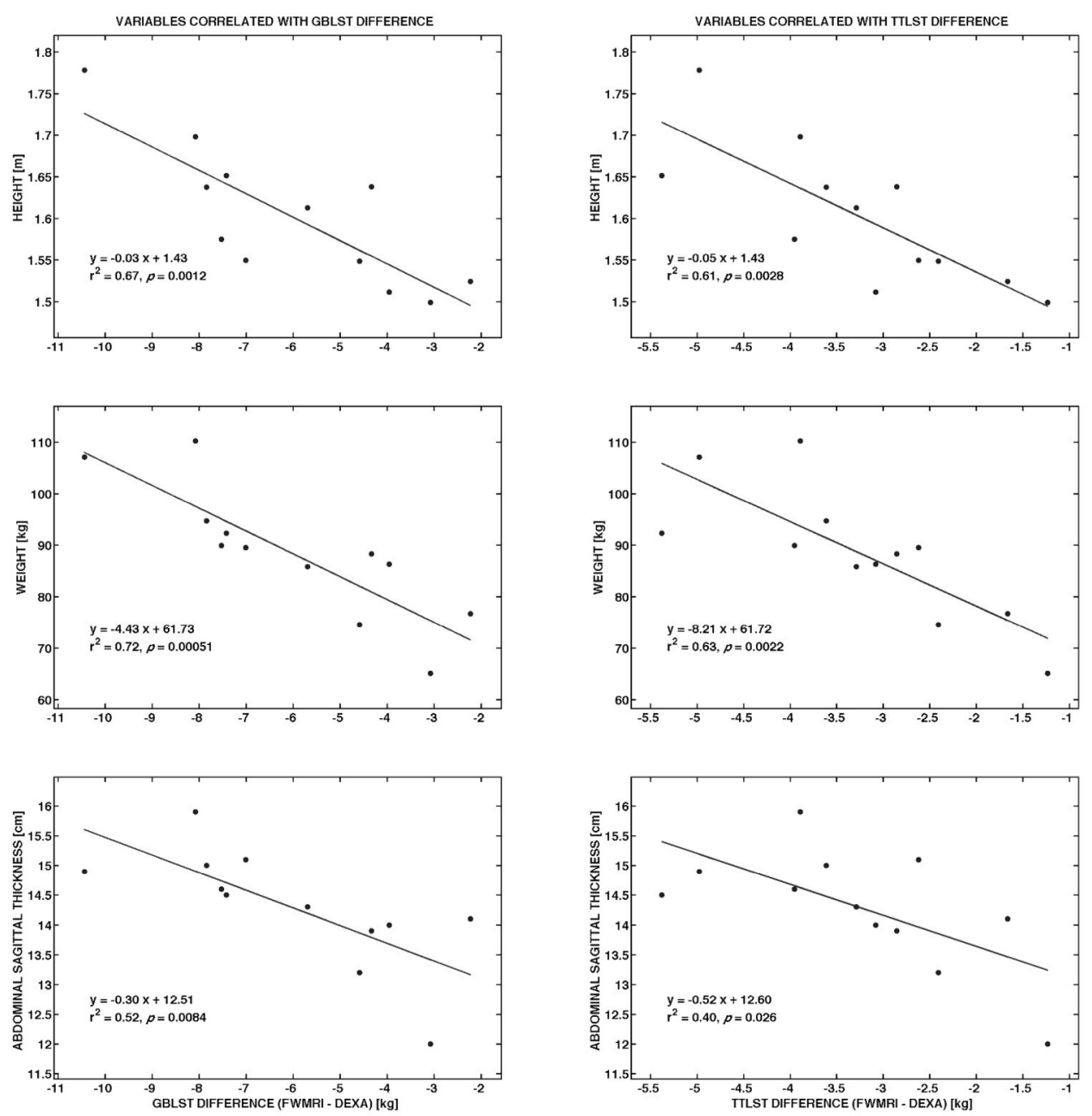

Figure 5.

Scatter plots and fitted linear trends of anthropometric variables (height, weight and DEXAderived abdominal sagittal thickness), which correlated significantly with the observed difference between FWMRI and DEXA for GBLST and TTLST. 


\section{Table 1}

Demographic characteristics of female subjects $(N=12)$.

\begin{tabular}{lcc}
\hline & Mean $\pm \mathbf{3 3}$ & Range \\
Age (years) & $36.7 \pm 9.0$ & $21.7-48.0$ \\
Height $(\mathrm{cm})$ & $160.2 \pm 7.9$ & $149.9-177.8$ \\
Weight $(\mathrm{kg})$ & $88.4 \pm 12.1$ & $65.1-110.2$ \\
Waist circumference $(\mathrm{cm})$ & $98.1 \pm 7.6$ & $80.0-107.5$ \\
Hip circumference $(\mathrm{cm})$ & $116.2 \pm 8.4$ & $104.0-135.5$ \\
Abdominal sagittal thickness $(\mathrm{cm})^{a}$ & $14.3 \pm 1.0$ & $12.0-15.9$ \\
BMI $\left(\mathrm{kg} / \mathrm{m}^{2}\right)^{b}$ & $34.3 \pm 2.8$ & $30.0-38.2$ \\
BAI $(\%)^{c}$ & $39.5 \pm 4.8$ & $29.0-46.7$ \\
\hline
\end{tabular}

${ }^{a}$ DEXA-derived measure defined as anterior-posterior soft tissue thickness

$b_{\mathrm{BMI}}=($ weight $[\mathrm{kg}]) /(\text { height }[\mathrm{m}])^{2}$

${ }^{c} \mathrm{BAI}=\left((\right.$ hip circumference $\left.[\mathrm{cm}]) /(\text { height }[\mathrm{m}])^{1.5}\right)-18$ 
Table 3

Correlations and differences between FWMRI and DEXA adipose and lean soft tissue masses.

\begin{tabular}{|c|c|c|c|c|}
\hline Tissue Depot ${ }^{a}$ & $\begin{array}{l}\text { Concordance Correlation Coefficient (95\% } \\
\text { CI) }\end{array}$ & Coefficient of Bias $b$ & Difference $^{c}(\mathbf{k g})^{d}$ & Percentage Difference \\
\hline GBAT & $0.978(0.923,0.994)$ & 0.996 & $0.1 \pm 1.5(P=0.75)$ & $0.2 \% \pm 4.0 \%$ \\
\hline TTAT & $0.802(0.468,0.935)$ & 0.884 & $-1.7 \pm 1.5(P=0.008)$ & $-10.4 \% \pm 9.1 \%$ \\
\hline GBLST & $0.629(0.324,0.816)$ & 0.646 & $-6.0 \pm 2.4(P=0.003)$ & $-12.8 \% \pm 3.8 \%$ \\
\hline TTLST & $0.400(0.120,0.620)$ & 0.423 & $-3.2 \pm 1.2(P=0.003)$ & $-20.9 \% \pm 5.2 \%$ \\
\hline
\end{tabular}

